\title{
Coarse Master Equations for Binding Kinetics of Amyloid Peptide Dimers
}

\section{Supplementary Information}

\section{Cathal T. Leahy ${ }^{1,2}$, Ronan D. Murphy ${ }^{1,2}$, Gerhard Hummer ${ }^{3}$,}

\section{Edina Rosta ${ }^{4}$, and Nicolae-Viorel Buchete ${ }^{1,2, *}$}

${ }^{1}$ School of Physics, University College Dublin, Belfield, Dublin 4, Ireland

${ }^{2}$ Complex and Adaptive Systems Laboratory, University College Dublin, Belfield, Dublin 4, Ireland

${ }^{3}$ Department of Theoretical Biophysics, Max Planck Institute of Biophysics, Max-von-Laue-

Straße 3, D-60438 Frankfurt am Main, Germany

${ }^{4}$ Department of Chemistry, King's College London, London SE1 1DB, United Kingdom

Date: June $24^{\text {th }}, 2016$

Email: buchete@ucd.ie

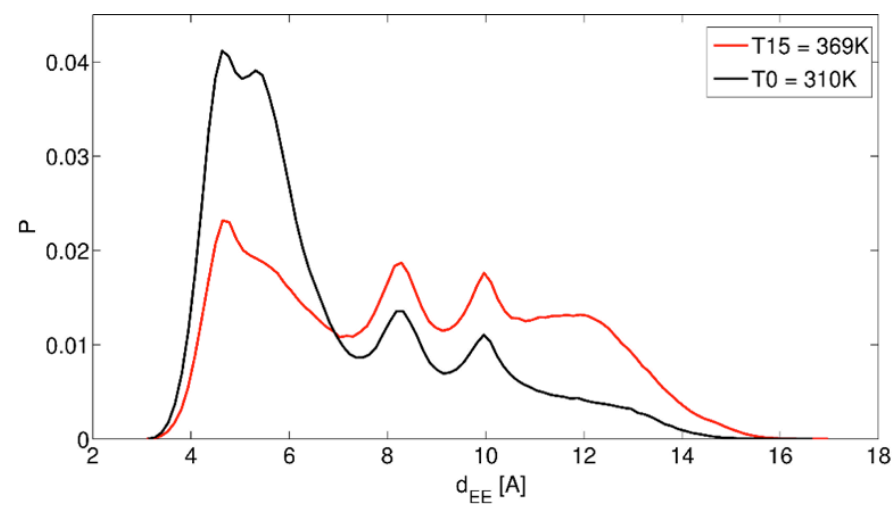

Figure S1: End-to-end distance $\left(d_{E E}\right)$ distributions for individual NNQQ peptides at the lowest temperature $\left(T_{0}=310 \mathrm{~K}\right.$, black line $)$, and at the highest simulation temperature $\left(T_{15}=369 \mathrm{~K}\right.$, red line) in the REMD simulations. 


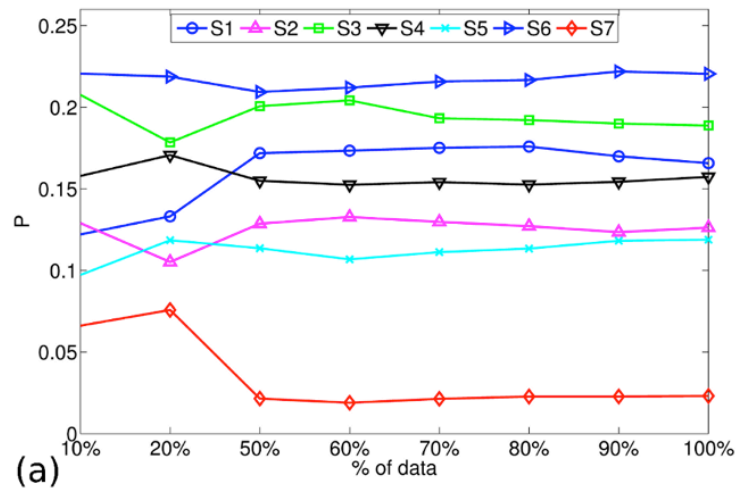

\section{$\mathrm{T} 0=310 \mathrm{~K}$}
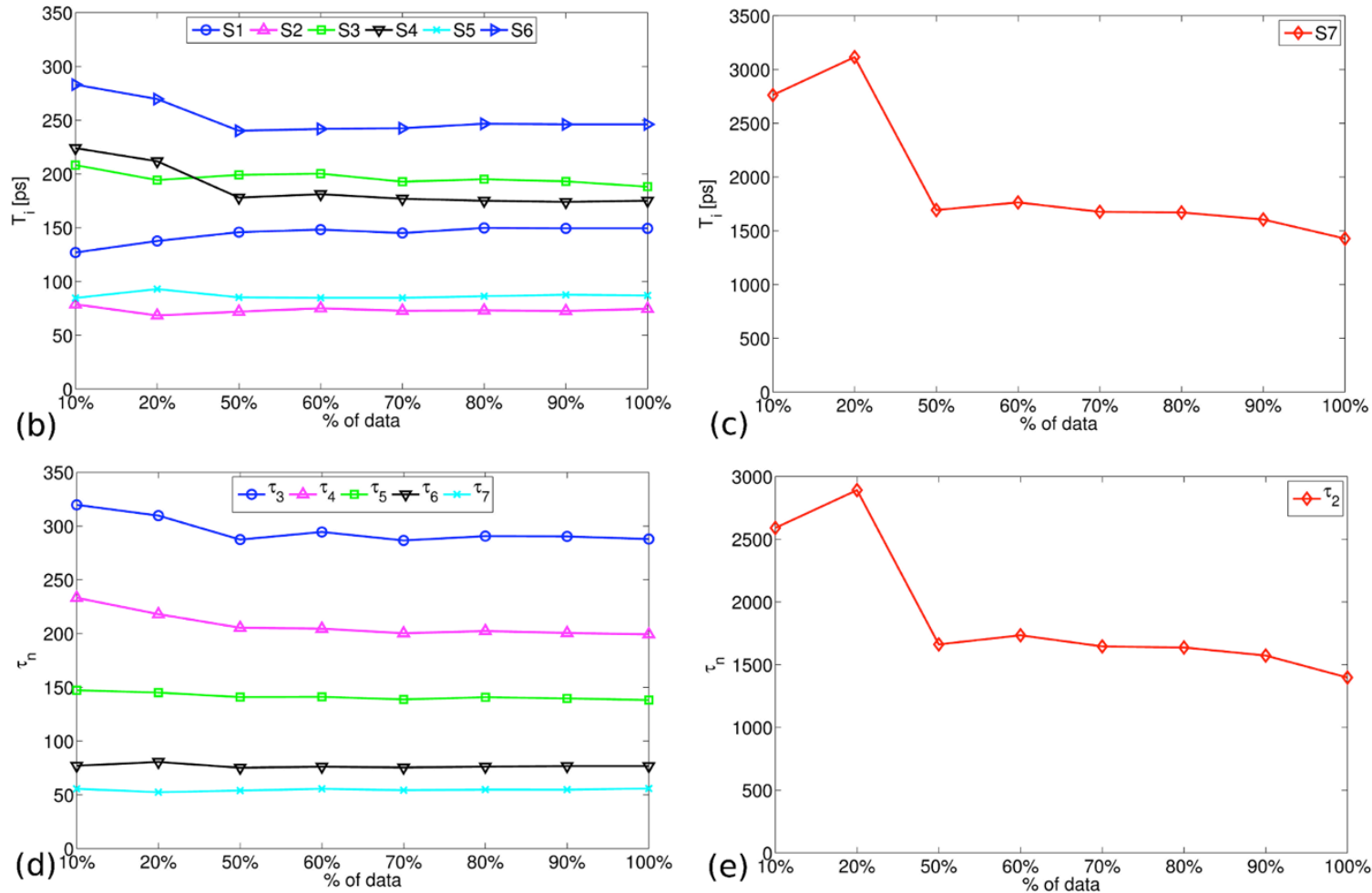

Figure S2: Convergence of the extracted kinetic model parameters, for the lowest temperature $T_{0}$ $(310 \mathrm{~K})$ in the replica exchange simulations. The values extracted are calculated by using various fractions of the amount of REMD data for: (a) the population of each state; (b, c) the lifetimes of each state (in ps, the life time of the dissociated state $S_{7}$ shown in (c)); and (d, e) the relaxation times of the system (in ps; the slowest relaxation time is shown in (e)). 


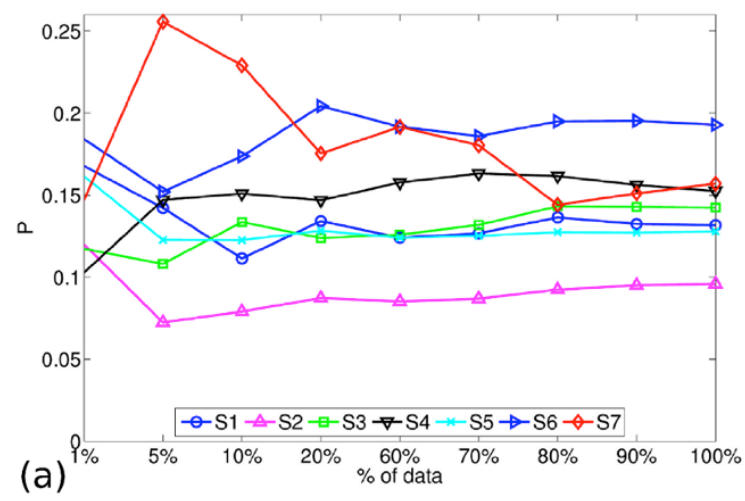

\section{$\mathrm{T} 15=369 \mathrm{~K}$}
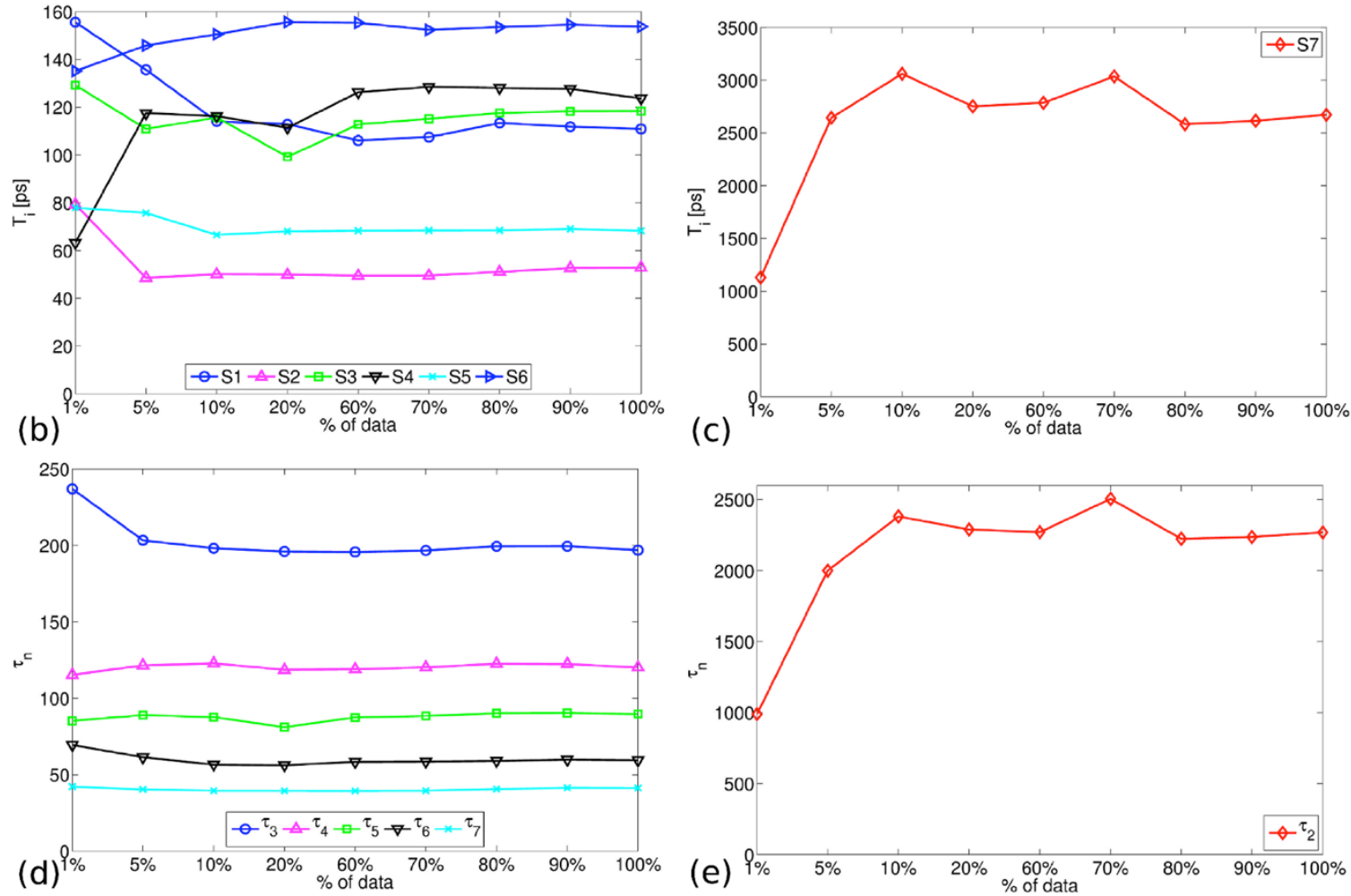

Figure S3: Convergence of the extracted kinetic model parameters, for the highest temperature $T_{15}(369 \mathrm{~K})$ in the replica exchange simulations. The values extracted are calculated by using various fractions of the amount of REMD data for: (a) the population of each state; $(b, c)$ the lifetimes of each state (in ps, the life time of the dissociated state S7 shown in (c)); and (d, e) the relaxation times of the system (in ps; the slowest relaxation time is shown in (e)). 

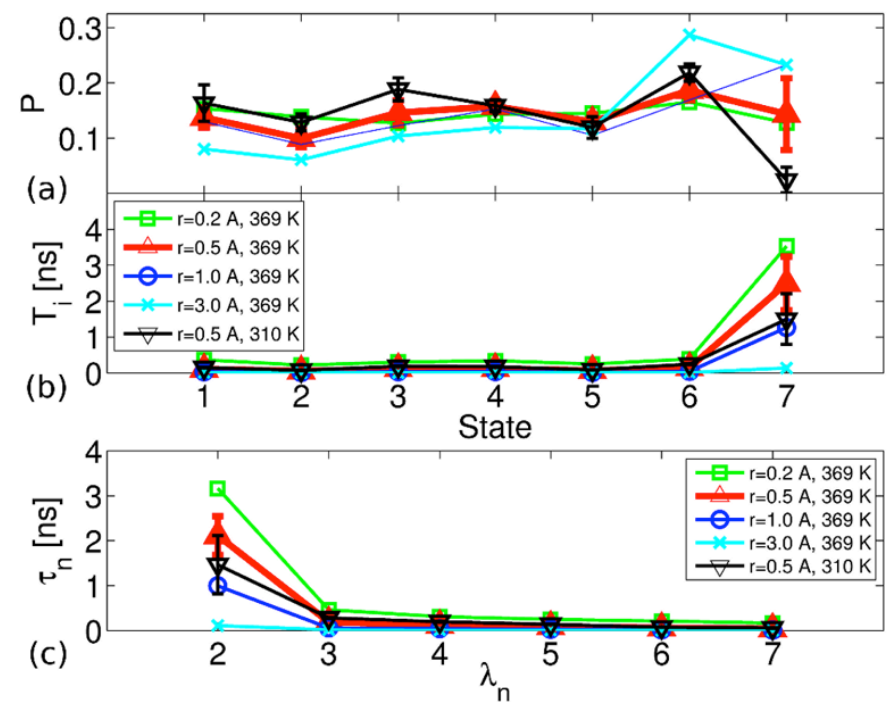

Figure S4: Dependence of the extracted kinetic model parameters on the quality of the state assignment method. Various values of the radius $(r)$ used to initially assign the conformational states around the probability maxima (see Fig. 1a in the main text) are used to calculate: (a) the populations, (b) lifetimes, and (c) relaxation times of this system. The initial value of $r=0.5 \AA$ (shown in black for $T_{0}=310 \mathrm{~K}$, and in red for $T_{15}=369 \mathrm{~K}$ ) is used for all remaining calculations. Note that T-trajectories capture the relevant physical dynamics. 

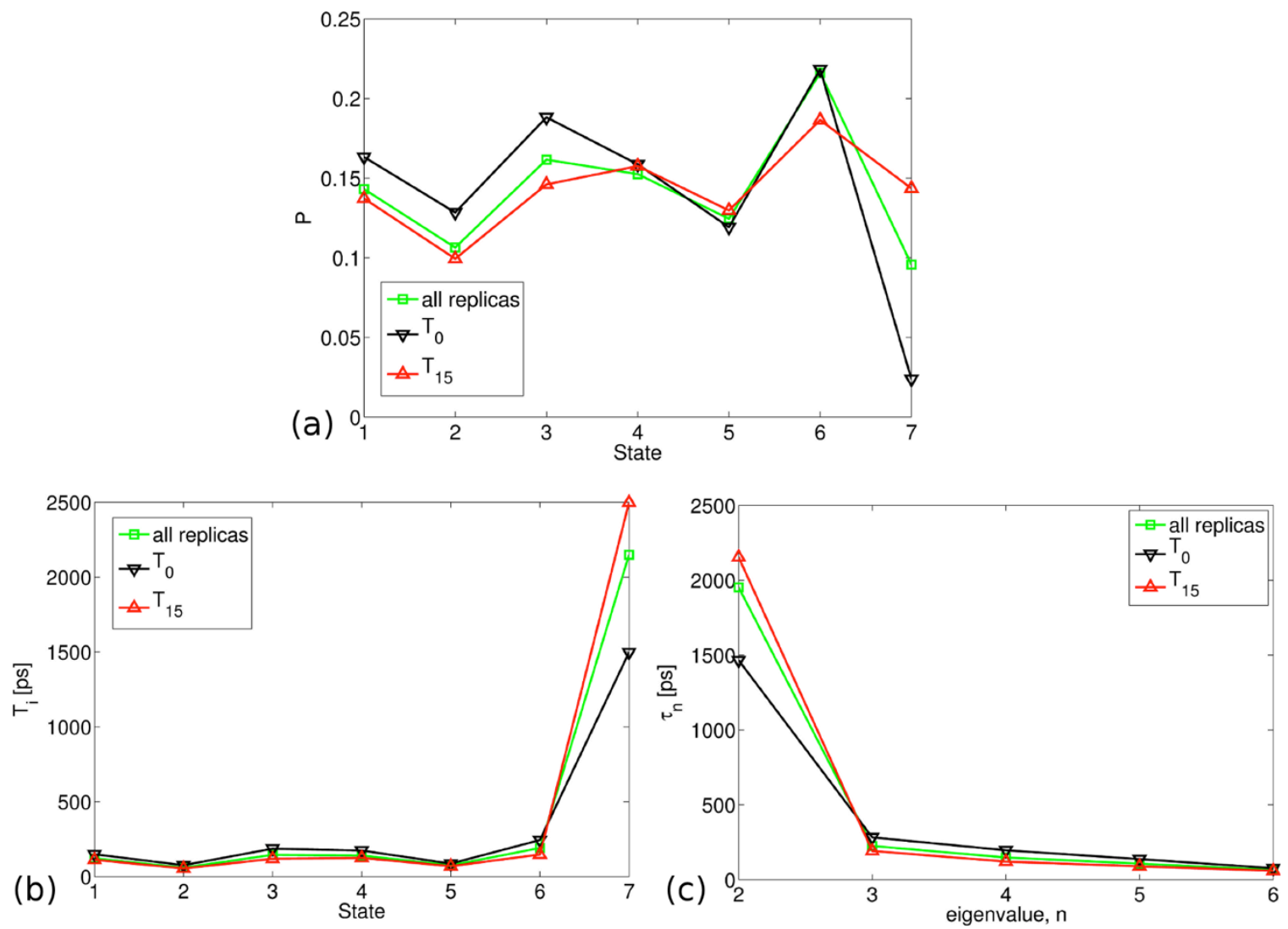

Figure S5: (a) Populations, (b) lifetimes and (c) relaxation times extracted from the REMD replica " $R$-trajectories" (green squares, corresponding to each replica evolving at various temperatures), and from the temperature " $T$-trajectories" (i.e., corresponding to REMD data at the highest (red) and the lowest (black) temperatures), respectively. 

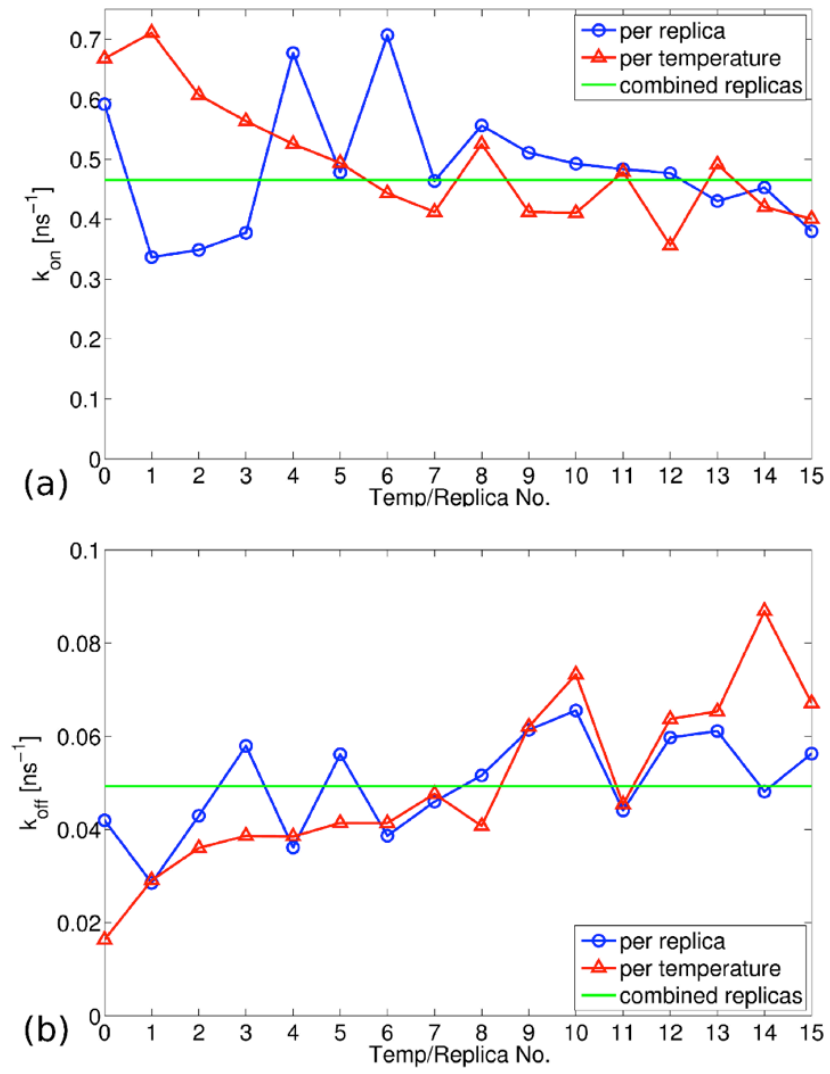

Figure S6: The rates of (a) association, $k_{o n}$, and (b) dissociation, $k_{\text {off }}$, as a function of the temperature (red triangles), and of each replica number (blue circles). The values extracted from the analysis of all the combined "replica" $R$-trajectories is shown in green. The temperatures used were spaced according to an optimized protocol at values 310.00, 313.69, 317.42, 321.18, $324.97,328.79$, 332.66, 336.55, 340.49, 344.46, 348.47, 352.51, 356.60, 360.72, 364.88, and $369.08 \mathrm{~K}$, respectively (see main text). 

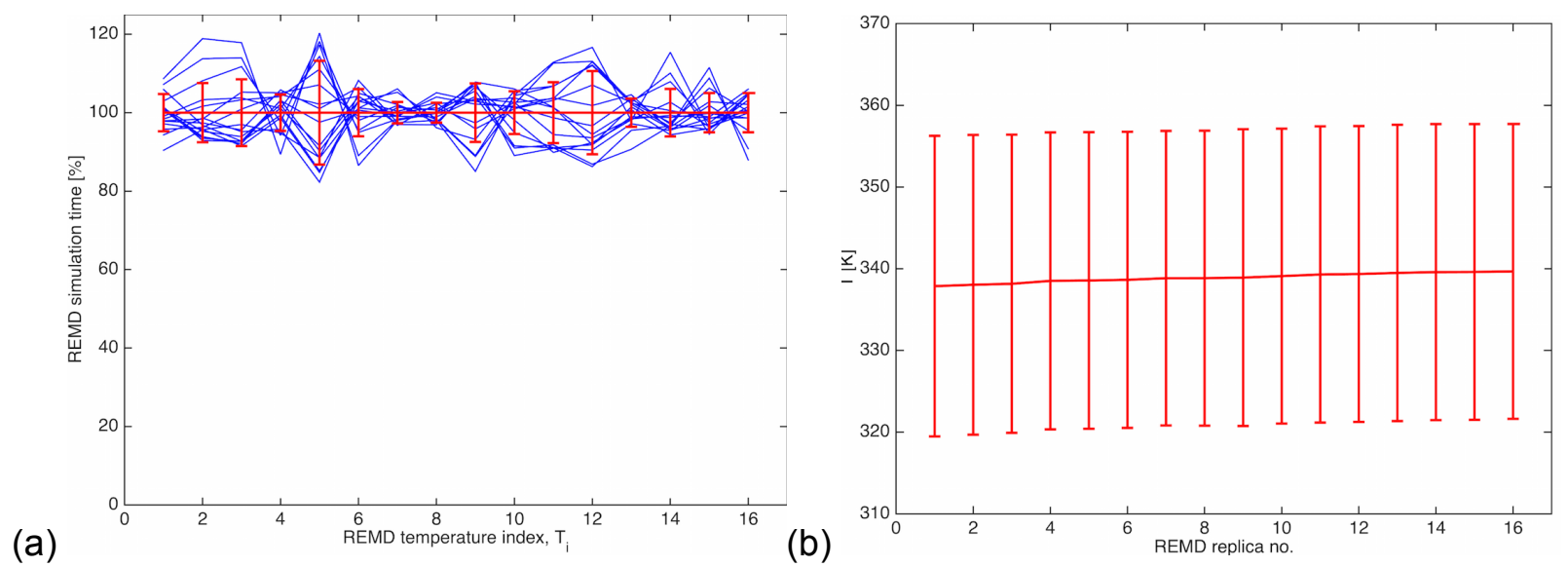

Figure S7: (a) The fraction of the total REMD simulation time spent by each of the 16 replicas (blue lines) at each temperature, $T_{i}$, with $i \in\{1,2, \ldots, 16\}$ being the temperature index (100\% corresponds to equal sampling of all temperatures). The temperatures used were spaced according to an optimized protocol at values $T_{1}=310.00 \mathrm{~K}, T_{2}=313.69 \mathrm{~K}, \ldots, T_{16}=369.08 \mathrm{~K}$, respectively (see main text and Fig. S6). The error bars (red) correspond to one standard deviations of the REMD simulation time of each replica at each temperature. The values shown here correspond to our first initial condition 1B (see main text and Fig. 1). (b) The corresponding averages and standard deviations (shown as error bars) for each replica. Note the very small increase in averages with the replica index, as replica 1 was initiated at the lowest temperature $(310 \mathrm{~K})$, and replica 16 at the highest $(369.08 \mathrm{~K})$. 